\title{
Singular Integral Equations with Monotone Nonlinearity in Complex Lebesgue Spaces
}

\author{
S. N. AskihaBov
}

\begin{abstract}
By methods of monotone operator theory, existence and uniqueness theorems are proved for some classes of nonlinear singular integral equations of Cauchy's type involving large nonlinearities in weighted complex Lebesgue spaces and also norm estimates of solutions are obtained.

Key words: Singular operators, nonlinear singular integral equations, method of monotone operators
\end{abstract}

AMS subject classification: 45005,35015

\section{Introduction}

There is a large literature on nonlinear singular integral equations of Hilbert's and Cauchy's type with (in some sense) small nonlinear terms (see [4] and the references therein). In recent years without smallness assumptions on the nonlinearities existence of solutions in real Lebesgue spaces $L_{p}$ is obtained for some classes of nonlinear equations with Hilbert and Cauchy kernel by means of the theory of monotone operators by mainly German and Soviet mathematicians (see the surveys $[1,6,13]$ ). In the present paper by methods of monotone operator theory existence and uniqueness theorems are proved for three different classes of nonlinear singular integral equations of Cauchy's type involving large nonlinearities in weighted complex Lebesgue spaces $\mathcal{L}_{p}(p)$ and also norm estimates of solutions are obtained.

\section{Preliminaries}

At first we state some known properties of the Cauchy singular integral operator and the basic theorems of monotone operator theory in complex Banach spaces, which are used in the sequel.

Let $\rho$ be a non-negative real measurable function on the whole real axis $\mathbb{R}$, which is almost everywhere finite and different from zero. Then $L_{p}(\rho), p>1$, is the Banach space of all complex-valued measurable functions $u$ on $R$ with finite norm $\|u\|=\left(\int_{R} \rho(x)|u(x)|^{P} d x\right)^{1 / P}$. We write $u \in L_{p}^{+}(\rho)$ if additionally $u$ is a non-negative function. For $\rho=1$ we simply write $L_{p}$ and $\|\cdot\|_{p}$, respectively. The dual space to $L_{p}(p)$ is the space $L_{q}\left(p^{1-q}\right)$ with $q=p /(p-1)$, the conjugate exponent to $p$, and norm $\|\cdot\|_{\text {. }}$.

We introduce the Cauchy singular integral operator (the so-called Hilbert transform)

$$
(\mathrm{S} u)(x)=\frac{1}{\pi} \int_{-\infty}^{+\infty} \frac{u(x)}{s-x} d s, x \in \mathbb{R} .
$$


The following basic characteristics are well known (see $[7,9]$ ):

$$
\|\mathbf{S} u\|_{2}=\|u\|_{2},(\mathbf{S} u, v)=-(u, \mathbf{S} v) \text { for all } u, v \in L_{2}
$$

$\|\mathbf{S} u\| \leq\|\mathbf{S}\|\|u\|$ for all $u \in L_{p}(p), p(x)=|x|^{\alpha},-1<\alpha<p-1$

where $(\cdot, \cdot)$ and $\|\cdot\|$ denote the usual scalar product and norm, respectively. From (1) it follows that $(\mathbf{S} u, u)=-(u, \mathbf{S} u)=-\overline{(\mathbf{S} u, u)}$ for all $u \in \zeta_{2}$ so that $\mathbf{S}$ is a positive operator in $\zeta_{2}$ since

$$
\operatorname{Re}(\mathbf{S} u, u)=0 \text { for all } u \in L_{2} \text {. }
$$

Let $X$ be a complex Banach space, $X^{*}$ the conjugate space of $X$, and $\langle\cdot, \cdot\rangle$ the pairing between $X^{*}$ and $X$. The following basic theorem of monotone operator theory ([5]; cf. also [3: Theorem of Browder and Minty]) is well known.

Theorem 1: Let $X$ be a reflexive separable complex Banach space and $\mathbf{A}: X \rightarrow X^{*}$ a monotone, hemicontinuous, coercive and bounded operator. Then the equation $\mathbf{A} u=f$ has a solution $u^{*} \in X$ for any $f \in X^{*}$. This solution is uniquely determined if $\mathbf{A}$ is strictly monotone.

This theorem implies (cf. [3,5]) the following

Corollary 1: Assume that A: $\mathcal{L}_{p}(\rho) \rightarrow \mathcal{L}_{q}\left(\rho^{1-q}\right), p>1$, is a strictly monotone, hemicontinuous and bounded operator. If there exists a real-valued function $\gamma=\gamma(t)$ of the non-negative argument $t$ with the property $\lim _{t \rightarrow \infty} \gamma(t)=+\infty$ such that $\operatorname{Re}\langle\mathbf{A} u, u\rangle \geq \gamma(\|u\|)\|u\|$ for all $u \epsilon$

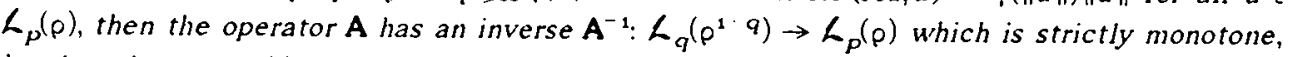
hemicontinuous and bounded.

Now suppose that $F(x, z): \mathbb{R} \times \mathbb{C} \rightarrow \mathbb{C}$ satisfies the Caratheodory conditions (i.e., $F(\cdot, z)$ is measurable for all $z \in \mathbb{C}$ and $F(x, \cdot)$ is continuous for almost all $x \in \mathbb{R})$ and $\operatorname{let}(\mathbf{F} u)(x)=F(x, u(x))$ be the corresponding Nemytski operator. Let us write out for the sake of reference convenience all the conditions used below on the function $F$ determining nonlinearity of the investigated equations. Namely, depending on the class of the investigated equations suppose that $F$ satisfies either the conditions (i) - (iii) or (iv) - (vi) $\left(d_{1}, \ldots, d_{4}\right.$ - positive constants):

(i) $|F(x, z)| \leq c(x)+d_{1} \rho(x)|z|^{p-1}$ for a.e. $x \in \mathbb{R}$ and all $z \in \mathbb{C}\left(c \in \mathcal{L}_{q}^{+}\left(\rho^{1-q}\right)\right)$.

(ii) $\operatorname{Re}\left\{\left(F\left(x, z_{1}\right)-F\left(x, z_{2}\right)\right) \overline{\left(z_{1}-z_{2}\right)}\right\} \geq 0$ for a.e. $x \in \mathbb{R}$ and all $z_{1}, z_{2} \in \mathbb{C}$.

(iii) $\operatorname{Re}\left\{(F(x, z) \bar{z}\} \geq d_{2} \rho(x)|z|^{P}-\mathcal{D}(x)\right.$ for a.e. $x \in \mathbb{R}$ and all $z \in \mathbb{C}\left(\mathcal{D} \in \mathcal{L}_{1}^{+}\right)$.

(iv) $|F(x, z)| \leq g(x)+d_{3}\left((\rho(x)){ }^{1}|z|\right)^{2 /(p-1)}$ for a.e. $x \in \mathbb{R}$ and all $z \in \mathbb{C}\left(g \in L_{p}^{+}(\rho)\right)$.

(v) $\operatorname{Re}\left\{\left(F\left(x, z_{1}\right)-F\left(x, z_{2}\right) \overline{\left(z_{1}-z_{2}\right)}\right\}>0\right.$ for a.e. $x \in \mathbb{R}$ and all $z_{1}, z_{2} \in \mathbb{C}$ such that $z_{1} \neq z_{2}$.

(vi) $\operatorname{Re}\left\{(F(x, z) \bar{z}\} \geq d_{1}\left((\rho(x))^{-1}|z|\right)^{1 /(p-1)}|z|-\mathcal{D}(x)\right.$ for a.e. $x \in \mathbb{R}$ and all $z \in \mathbb{C}\left(\mathcal{O} \in L_{1}^{+}\right)$.

Let us notice that if the conditions (i) - (iii) are fulfilled, then the Nemytski operator $F$, associated with the function $F(x, z)$, is a bounded and continuous, monotone, coercive mapping from the whole space $\mathcal{L}_{p}(\rho)$ into $\mathcal{L}_{q}\left(\rho^{1-q}\right)$ (cf. $\left.[4,14]\right)$ and if the conditions (iv) - (vi) are fulfilled, then the operator $F$ is a bounded and continuous, strictly monotone, coercive mapping from the whole space $\mathcal{L}_{q}\left(\rho^{1-q}\right)$ into $\mathcal{L}_{p}(\rho)$. 


\section{On the positiveness of some singular operators in weighted complex Lebesgue spaces}

As is well known, the singular operator $\mathbf{S}$ doesn't act in general from $L_{p}$ into $L_{q}$ when $p \neq q$, and therefore it has the property of positiveness only in the case $p=q=2$, as it can be seen from (3). In connection with this the lemmas used below are of interest in investigating the corresponding nonlinear singular integral equations.

Lemma 1: Let be $p \geq 2$,

$$
b, w \in K_{2 p /(p-2)}\left(\rho^{2 /(2-p)}\right) \text { as } p>2 \text { and } b / \sqrt{p}, w / \sqrt{\rho} \in K_{\infty} \text { as } p=2 \text {. }
$$

Then the singular operator

$$
(\mathbf{Q} u)(x)=\frac{1}{\pi} \int_{-\infty}^{+\infty} \frac{(\overline{b(x)} w(s)+b(s) \overline{w(x)}) u(s)}{s-x} d s
$$

is a bounded and positive mapping from the whole space $\zeta_{p}(\rho)$ into $L_{q}\left(\rho^{1-q)}\right.$. More precisely, we have

$$
\left\|Q_{u}\right\|_{*} \leq 2\left\|\rho^{-1 / p} b\right\|_{2 p /(p-2)}\left\|\rho^{-1 / p} w\right\|_{2 p /(p-2)}\|u\| \text { and } \operatorname{Re}\langle Q u, u\rangle=0 \forall u \in L_{p}(\rho) .
$$

Proof: Using Hölder's inequality, we have $\|w u\|_{2} \leq\left\|p^{-1 / p} w\right\|_{2 p /(p-2)}\|u\|$, which implies that $w u \in \mathcal{L}_{2}$. Then, by (1), we get $\|S(w u)\|_{2} \leq\left\|p^{-1 / p_{w}}\right\|_{2 p /(p-2)}\|u\|$. Hence

$$
\|b \mathbf{S}(w u)\| . s\left\|p^{-1 / p} b\right\|_{2 p /(p-2)} \| p^{-1 / p_{W}\left\|_{2 p /(p-2)}\right\| u \| .}
$$

Analogously we obtain the estimate

$$
\|\bar{W} \mathbf{S}(b u)\| . s\left\|\rho^{-1 / p} b\right\|_{2 p /(p-2)}\left\|\rho^{-1 / p} w\right\|_{2 p /(p-2)}\|u\| .
$$

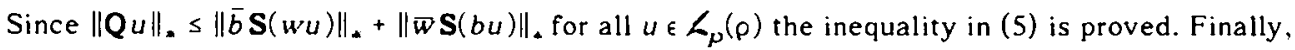
using (1) we have

$$
\langle\mathbf{Q} u, u\rangle=-2 \mathrm{i} \operatorname{Im}(w u, \mathbf{S}(b u)) \text { for all } u \in \zeta_{p}(\rho)
$$

so that the equality in (5) is proved

Corollary 2: Let be $p \geq 2$ and

$$
c(\rho):= \begin{cases}\left(\int_{-\infty}^{+\infty}(\rho(x))^{2 /(2-p)} d x\right)^{(p-2) / 2 p}<\infty & \text { as } p>2 \\ \sup _{-\infty<x<+\infty}(\rho(x))^{-1 / 2}<\infty & \text { as } p=2 .\end{cases}
$$

Then $\mathbf{S}: \zeta_{p}(\rho) \rightarrow L_{q}\left(p^{1-q)}\right.$ is a bounded and positive operator, i.e. $\|\mathbf{S} u\|_{\text {. }} \leq c^{2}(\rho)\|u\|$ and $\operatorname{Re}\langle\mathbf{S} u, u\rangle=0$ for all $u \in \zeta_{p}(\rho)$.

We note that, under the assumptions of Corollary 2 , we have $h_{p}(\rho) \subset L_{2} \subset L_{q}\left(\rho^{1-q)}\right.$. Analogously the following lemma is obtained.

Lemma 1: Let be $1<p \leq 2$,

$$
b, w \in L_{2 p /(2-p)}\left(p^{2 /(2-p)}\right) \text { as } 1<p<2 \text { and } b \sqrt{\rho} \text {, w } \sqrt{\rho} \in \mathcal{L}_{m} \text { as } p=2 \text {. }
$$


Then $\mathrm{Q}: \boldsymbol{L}_{\boldsymbol{q}}\left(\rho^{1-9}\right) \rightarrow L_{p}(\rho)$ is a bounded and positive operator, i.e.

$\|Q v\| \leq 2\left\|p^{1 / p} b\right\|_{2 p /(2-p)}\left\|p^{1 / p_{W}}\right\|_{2 p /(2-p)}\|v\|_{*}$ and $\operatorname{Re}\langle Q v, v\rangle=0 \forall v \in L_{q}\left(\rho^{1-q)}\right.$.

There holds the following

Lemma 2: Let be $p \geq 2$ and $p(x)=|x|^{\alpha}$, where $-1<\alpha<p-1$. If

$w \in L_{p /(p-2)}\left(p^{2 /(2-p)}\right)$ as $p>2$ and $w / p \in \mathcal{L}_{\infty}$ as $p=2$,

then the singular operator

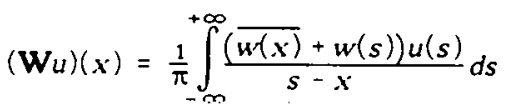

is a bounded and positive mapping from the whole space $h_{p}(p)$ into $L_{q}\left(p^{1-q}\right)$, i.e.

$\left\|W_{u}\right\|_{*} \leq 2 v(p, \alpha)\left\|_{p}^{-2 / p_{W} \|_{p} /(p-2)}\right\| u \|$ and $\operatorname{Re}\langle\mathbf{W} u, u\rangle=0$ for all $u \in L_{p}(\rho)$

where (see [7])

$$
v(p, \alpha)= \begin{cases}\operatorname{ctg}(\pi(1+\alpha) / 2 p) & \text { as }-1<\alpha<0 \\ \operatorname{ctg}(\pi / 2 p) & \text { as } 0 \leq \alpha \leq p-2 \\ \operatorname{ctg}(\pi(p-1-\alpha) / 2 p) & \text { as } p-2<\alpha<p-1 .\end{cases}
$$

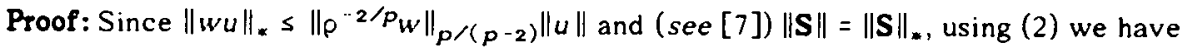

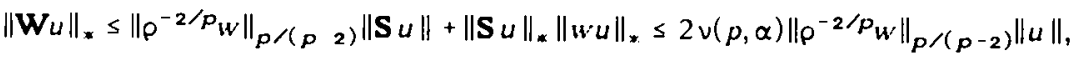
so that the inequality in (9) is proved. Finally, using (1) we have $\langle\mathbf{W} u, u$ ) $=2 \mathrm{i} \operatorname{Im}\langle\mathbf{S} u, w u\rangle$ (cf. (6)) so that the equality in (9) is proved

Analogously the following lemma is obtained.

Lemma 2: Let be $1<p \leq 2$ and $\rho(x)=|x|^{\alpha}$, where $-1<\alpha<p-1$. If

$w \in \mathcal{L}_{p /(2-p)}\left(\rho^{2 /(2-p)}\right)$ as $1<p<2$ and $w \rho \in \mathcal{L}_{\infty}$ as $p=2$,

then $\mathbf{W}: \zeta_{q}\left(p^{1-q}\right) \rightarrow \zeta_{p}(p)$ is a bounded and positive operator, i.e.

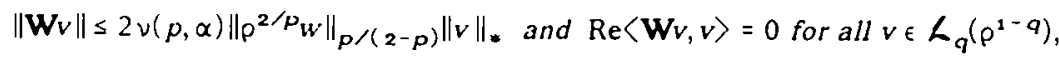
where $(\sec [7]) v(p, \alpha)=v(q, \alpha(1-q))$ is determined by formula $(10)$.

\section{Existence and uniqueness theorems}

Let us first consider some equations which are simpler for investigation.

Theorem 2: Let be $p \geq 2, b$ and $w$ satisfy the condition (4) and $a$ be a non-negative a.e. different from zero function on $\mathbb{R}$ satisfying the condition (8). If the function $F(x, z)$ satisfies the conditions (i) - (iii), then the equation 


$$
A u:=\lambda_{1} a u+\lambda_{2} Q u+\lambda_{3} F u=f
$$

has a solution $u^{*} \in L_{p}(\rho)$ for any function $f \in L_{q}\left(\rho^{1-q}\right)$ and for any $\lambda_{1} \in \mathbb{C}$ and $\lambda_{2}, \lambda_{3} \in \mathbb{R}$ such that $\lambda_{3} \operatorname{Re} \lambda_{1} \geq 0, \lambda_{3} \neq 0$. Moreover, if additionally $\mathcal{D}=0$, then the inequality

$$
\left\|u^{*}\right\| \leq\left(d_{2}^{-1} \lambda_{3}^{-1}\|f\|_{*}\right)^{1 /(p-1)}
$$

holds. The solution $u^{*}$ is uniquely determined if either $\lambda_{3} \operatorname{Re} \lambda_{1}>0$ or the condition (v) is fulfilled.

Proof: By a shift we can always assume that $\operatorname{Re} \lambda_{1} \geq 0$ and $\lambda_{3}>0$. From the conditions (i)

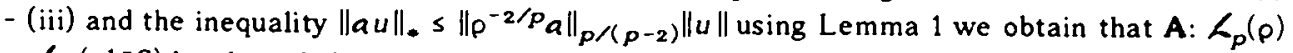
$\rightarrow L_{q}\left(p^{1-q}\right)$ is a bounded, continuous, monotone and coercive operator. Hence, by Theorem 1 , we infer that the equation (12) has a solution $u^{*} \in \zeta_{p}(\rho)$ and this solution is uniquely determined if either $\operatorname{Re} \lambda_{1}>0$ or the condition $(v)$ is fulfilled. Finally, we note that for $D=0$

$$
\lambda_{3} d_{2}\left\|u^{*}\right\|^{P} \leq \operatorname{Re}\left\langle\boldsymbol{A} u^{*}, u^{*}\right\rangle=\operatorname{Re}\left\langle f, u^{*}\right\rangle \leq\|f\|_{*}\|u\|_{*}
$$

so that the inequality (13) is true

Analogously, by using Lemma 2 instead of Lemma 1 there is proved the following

Theorem 2': Let be $p \geq 2$ and $p(x)=|x|^{\alpha}$, where $-1<\alpha<p-1$. Let $w$ satisfy the condition (8) and let $a, F, f, \lambda_{1}, \lambda_{2}, \lambda_{3}$ satisfy the conditions of Theorem 2 . Then the equation

$$
\lambda_{1} a(x) u(x)+\lambda_{2}(W u)(x)+\lambda_{3} F(x, u(x))=f(x)
$$

has a solution $u^{*} \in \zeta_{p}(p)$. Moreover, if additionally $\mathcal{O}=0$, then the inequality (13) holds. The solution $u^{*}$ is uniquely determined if either $\lambda_{3} \operatorname{Re} \lambda_{1}>0$ or the condition $(v)$ is fulfilled.

Remark 1: The simplest example of a function $F$ satisfying the conditions (i) - (iii), (v) is $F(x, z)=\rho(x) z|z|^{p-2}$, where $p$ is an even number.

We now consider the general nonlinear singular integral equation of Hammerstein's type. There holds the following

Theorem 3: Let be $1<p \leq 2$ and let $b, w$ satisfy the condition (7), $F$ the conditions (i), (iii) and $(v)$. Then the equation

$$
u+\lambda Q \mathbf{Q F} u=f
$$

has a unique solution $u^{*} \in \zeta_{p}(\rho)$ for any $f \in L_{p}(\rho)$ and each fixed $\lambda \in \mathbb{R}$. Moreover, if additionally $c=0$ and $\mathcal{D}=0$, then the inequality

$$
\left\|u^{*}\right\| \leq d_{1} d_{2}^{-1}\|f\|
$$

holds, and if instead of it the condition

(vii) $\operatorname{Re}\left\{(F(x, z) \bar{z}\} \geq d_{5}\left((\rho(x))^{-1}|F(x, z)|^{P}\right)^{1 /(p-1)}\right.$ for a.e. $x \in \mathbb{R}$ and all $z \in \mathbb{C}\left(d_{s}>0\right)$ is fulfilled, then there holds the inequality

$$
\left\|u^{*}-f\right\| \leq 2|\lambda|\left\|\rho^{1 / p} b\right\|_{2 p /(2-p)}\left\|\rho^{1 / p_{w}}\right\|_{2 p /(2-p)}\left(d_{5}^{-1}\|f\|\right)^{p-1} .
$$

6 Analysis. Bd. 11. Heft 1 (1992) 
Proof: From the conditions (i), (iii) and (v), by Corollary 1 we infer that there exists the inverse operator $F^{-1}: L_{p}(p) \rightarrow L_{q}\left(\rho^{1-q}\right)$ and $F^{-1}$ is a strictly monotone, hemicontinuous and bounded operator. We shall prove that $F^{-1}$ is a coercive operator. Let $\psi \in \mathcal{L}_{q}\left(\rho^{1-q}\right)$ and $F^{-1} \psi=$ $\varphi$. By condition (i), we have

$$
\|\Psi\|_{*}=\|\mathbf{F} \varphi\|_{*} \leq\|c\|_{*}+d_{1}\|\varphi\|^{p-1}
$$

so that $\|\varphi\| \rightarrow \infty$ if $\|\psi\|_{\bullet} \rightarrow \infty$. Hence

$$
\frac{\operatorname{Re}\left\langle\mathbf{F}^{-1} \dot{\psi}, \psi\right\rangle}{\|\psi\|_{*}} \geq \frac{d_{2}\|\varphi\| P-\|\mathcal{D}\|_{1}}{\|c\|_{*}+d_{1}\|\varphi\|^{P^{-1}}} \rightarrow+\infty \text { as }\|\psi\|_{*} \rightarrow \infty,
$$

i.e. $\mathbf{F}^{-1}$ is a coercive operator.

We now consider the auxiliary equation

$\Phi_{v}=f$, where $\Phi_{v}:=\mathbf{F}^{-1} v+\lambda Q_{v}$.

It is easy to see that if $v^{*} \in L_{q}\left(p^{1-q}\right)$ is a solution of (18), then $u^{*}=\mathbf{F}^{-1} v^{*} \in L_{p}(p)$ is a solution of (14). Since $\Phi: L_{q}\left(\rho^{1-q)} \rightarrow L_{p}(\rho)\right.$ and $\Phi$ is a strictly monotone, hemicontinuous, coercive and

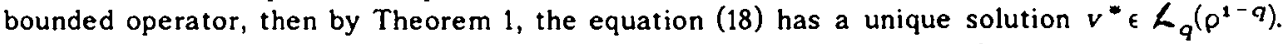
Hence, the equation (14) has a unique (by condition (iv)) solution $u^{*} \in \zeta_{p}(p)$.

We now prove the inequality (15). Using Lemma $1^{\prime}$ and the conditions (i), (iii) (with $c=0$ and $\mathcal{D}=0)$, by (17), we have

$$
d_{2}\left\|u^{*}\right\|^{P} \leq \operatorname{Re}\left\langle u^{*}, \mathrm{~F} u^{*}\right\rangle=\operatorname{Re}\left\langle f, F u^{*}\right\rangle \leq d_{1}\|f\|\left\|u^{*}\right\|^{p-1}
$$

so that (15) is true. Finally, using Lemma 1', we have

$$
\left\|u^{*}-f\right\| \leq 2|\lambda|\left\|\rho^{1 / p} b\right\|_{2 p /(2-p)}\left\|\rho^{1 / p} W\right\|_{2 p /(2-p)}\left\|\mathrm{F} u^{*}\right\|_{*} .
$$

Since $\operatorname{Re}\left\langle u^{*}, \mathbf{F} u^{*}\right\rangle \leq\|f\|\left\|\mathbf{F} u^{*}\right\|_{*}$ and, by condition (vii), $\operatorname{Re}\left\langle u^{*}, \mathbf{F} u^{*}\right\rangle \geq d_{s}\left\|\mathbf{F} u^{*}\right\|_{*}^{q}$, we have $\left\|\mathrm{F} u^{*}\right\|_{*} s\left(d_{5}^{-1}\|f\|\right)^{1 /(q-1)}$. Hence, by (19), the estimate (16) is true

Analogously, using Lemma 2', we can prove the following

Theorem 3': Let be $1<p \leq 2$ and $\rho(x)=|x|^{\alpha}$, where $-1<\alpha<p-1$. Let $w$ satisfy the condition (11) and $F, f$ and $\lambda$ the conditions of Theorem 3. Then the equation $u+\lambda W F u=f$ has $a$ uinque solution $u^{*} \in \mathcal{L}_{p}(p)$. Moreover, if additionally $c=D=0$, then the inequality (15) holds, and if instead of $i t$ the condition (vii) is fulfilled, then the inequality

$$
\left\|u^{*}-f\right\| \leq 2 v(\rho, \alpha)\left\|\rho^{2 / P_{w}}\right\|_{p /(2-p)}\left(d_{s}^{-1}\|f\|\right)^{p^{-1}}
$$

holds, where $v(p, \alpha)$ is determined in Lemma $2^{\prime}$.

Remark 2: Equations of types (12) and (14), where the role of the operator $Q$ is played by other singular operators were considered in [8], when $p=1$.

Let us consider the corresponding cases that a singular operator enters the equation nonlinearly.

Theorem 4: Let be $\rho 22$ and let b, w satisfy the condition (4), $F$ the conditions (iv) - (vi). Then the equation 
$u+\lambda \mathrm{FQ} u=f$

has a unique solution $u^{*} \in \zeta_{p}(\rho)$ for any $f \in L_{p}(\rho)$ and each fixed $\lambda \in \mathbb{R}$. Moreover, if additionally $g=0$ and $\mathcal{D}=0$, then there holds the inequalities

$$
\begin{aligned}
& \left\|u^{*}-f\right\| \leq d_{3} d_{4}^{-1}\|f\| \\
& \left\|u^{*}-f\right\| \leq\left(2 d_{3}^{P} d_{4}^{-1}\left\|\rho^{-1 / p} b\right\|_{2 p /(p-2)}\left\|\rho^{-1 / P} W\right\|_{2 p /(p-2)}\|f\|\right)^{1 /\left(p^{-1}\right)} .
\end{aligned}
$$

Proof: From the conditions of the theorem we infer that

$$
Q: L_{p}(p) \rightarrow L_{q}\left(\rho^{1-q)} \text { and } \mathbf{F}: L_{q}\left(\rho^{1-q)} \rightarrow L_{p}(p)\right.\right.
$$

and that $F$ is a strictly monotone, hemicontinuous, coercive and bounded operator. Hence, by Corollary 1 , the operator $F$ has an inverse $F^{-1}: L_{p}(\rho) \rightarrow L_{q}\left(p^{1-q}\right)$ and $F^{-1}$ is a strictly monotone, hemicontinuous, coercive ( $c f$. the proof of Theorem 3 ) and bounded operator. We introduce $v=\lambda^{-1}(f-u)$ as a new unknown and apply $\mathbf{F}^{-1}$ to both sides of $(20)$. Then we obtain the equation

$$
\Phi v=0, \text { where } \Phi v:=\mathbf{F}^{-1} v+\lambda \mathbf{Q} v-\mathbf{Q} f
$$

Since $\Phi: L_{p}(\rho) \rightarrow L_{q}\left(p^{1-q}\right)$ and $\Phi$ is a strictly monotone, hemicontinuous, coercive and bounded operator, then, by Theorem 1 , the equation (23) has a unique solution $v^{*} \in \zeta_{p}(\rho)$. Hence, the equation (20) has a unique (by condition (v)) solution $u^{*}=f-\lambda v^{*} \in \zeta_{p}(p)$.

We now prove the inequality (22). Let $\psi=\mathbf{F}^{-1} v^{*}$. Since $\mathbf{F}^{-1} v^{*}+\lambda \mathbf{Q}_{v^{*}}=\mathbf{Q} f$ and $g=0, \boldsymbol{D}=$ 0 , then, by (iv), (vi) and (5),

$$
\begin{aligned}
d_{1}\|\psi\|_{*} & \leq \operatorname{Re}\langle\mathbf{F} \psi, \psi\rangle=\operatorname{Re}\left\langle v^{*}, \mathbf{F}^{-1} v^{*}+\lambda Q v^{*}\right\rangle \\
& \leq 2 d_{3}\left\|p^{-1 / p} b\right\|_{2 p /(p-2)}\left\|p^{-1 / p} w\right\|_{2 p /(p-2)}\|f\|\|\psi\|_{*}^{q^{-1}}
\end{aligned}
$$

and $\left\|u^{*}-f\right\|=|\lambda|\left\|v^{*}\right\| s|\lambda| d_{3}\|\psi\|_{*^{-1}}^{q}$ so that the inequality (22) is true. The proof of the inequality (21) is similar to that of (15)

Analogously, using Lemma 2 , one gets the following

Theorem 4': Let be $p \geq 2, \rho(x)=|x|^{\alpha}$, where $-1<\alpha<p-1$, and $w$ satisfy the condition (8), $F, f$ and $\lambda$ the conditions of Theorem 4. Then the equation $u+\lambda F W u=f$ has a unique solution $u^{*} \dot{\epsilon} \zeta_{p}(\rho)$. Moreover, if additionally $g=0$ and $\mathcal{D}=0$, then the inequalities (21) and

$$
\left\|u^{*}-f\right\| \leq|\lambda|\left(2 d_{3}^{P} d_{4}^{-1} v(p, \alpha) \|_{\left.\rho^{-2 / p} w\left\|_{p /(p-2)}\right\| f \|\right)^{1 /(p-1)}}\right.
$$

hold: where $v(p, \alpha)$ is defined in $(10)$.

\section{REFERENCES}

[1] ASKHABOV, S.N.: Singular integral equations with monotone nonlinearity (Russian). Dep. VINITI Akad. Nauk SSSR 04.12.1989, N7198-V89. Grozny 1989, 1 - 75.

L2」 FRIEDMAN. M.J.: Mathematical study of the nonlinear singular integral magnetic field equation. SIAM J. Appl. Math. 39 (1980), 14 - 20.

[3] GヘJEWSKI,H., GRÖCER, K., and K. ZヘCHARIAS: Nichtlineare Operatorgleichungen und Operatordifferentialgleichungen. Berlin: Akademie - Verlag 1974. 
[4] GUSEINOV. A.I., and Kh. Sh. MUKHTAROV: Introduction to the Theory of Nonlinear Singular Integral Equations (Russian). Moscow: Nauka 1980.

[5] DUBINSKI, Yu.A.: Nonlinear Elliptic and Parabolic Equations (Russian). Itogi Nauki i Tekhniki: Sovrem. probl. mat. Akad. Nauk SSSR 9 (1976), 3 - 130

[6] KOSEL, U., and L.v. WOLFERSDORF: Nichtlineare Integralgleichungen. Sem. Anal.: Oper. Equ. Num. Anal. (Berlin) 1986, 93 - 128.

[7] KRUPNIK, N.Ya.: Banach Algebras with Symbol and Singular Integral Operators (Rus sian). Kishinev: Shtiintsa 1984.

[8] M^GOMEDOV, G.M., and Kh. B. KHANIK^LOV: Solvability theorems of nonlinear equa tions of the second kind and some applications (Russian). Dok1. Akad. Nauk SSSR 270 (1983), 1051 - 1053.

[9] NERI, U.: Singular Integrals. Lect. Notes Math 200 (1971),

[10] v. WOLFERSDORF. L.: Monotonicity Methods for nonlinear singular integral and integro -differential equations. ZAMM 63 (1983), 249 - 259.

[11] v. WOLFERSDORF, L.: On the theory of nonlinear singular integral equations of Cauchy type. Math. Meth. Appl. Sci. 7 (1985), 493 - 517.

L12 J v. WOLFERSDORF, L.: A class of nonlinear singular integral and integro-differential equations with Hilbert kernel. Z. Anal. Anw. 4 (1985), 385401.

[13] $v$. WOLFERSDORF, L.: Some recent developments in the theory of nonlinear singular integral equations. Z. Anal. Anw. 6 (1987), 83 - 92.

[14] ZEIDLER, E.: Vorlesungen über Nichtlineare Funktionalanalysis. Vol. II: Monotone Operatoren (Teubner-Texte zur Mathematik: Vol.9). Leipzig: B. C. Teubner Verlagsges. 1977.

Received 02.11.1990; in revised form 11.08.1991

Dr. Sultan Nazhmudinovich Askhabov

Checheno-Ingush State University

Department of Mathematics

u1. A. Sheripova 32

USSR 364907 Grozny 